\title{
Algebraic Complete Integrability of the Bloch-Iserles System
}

\section{Vasile Brînzănescu ${ }^{1}$ and Tudor S. Ratiu ${ }^{2}$}

1"Simion Stoilow" Institute of Mathematics of the Romanian Academy, P.O. Box 1-764, 014700 Bucharest, Romania and ${ }^{2}$ Section de Mathématiques and Bernoulli Center, École Polytechnique Fédérale de Lausanne, CH-1015 Lausanne, Switzerland

Correspondence to be sent to: e-mail: tudor.ratiu@epfl.ch

The goal of this paper is the proof of the algebraic complete integrability of the BlochIserles Hamiltonian system [5]. This result was conjectured in [4], based on its validity in certain special cases.

\section{Introduction}

For a given skew symmetric real $n \times n$ matrix $N$, the bracket $[X, Y]_{N}=X N Y-Y N X$ defines a Lie algebra structure on the $\operatorname{space} \operatorname{Sym}(n, N)$ of real symmetric $n \times n$ matrices; this type of bracket has appeared before in [12] in the study of the integrability of the Euler equations via the argument shift method and in the construction of the sectional operators as well as in [9] where it was used to produce the second Hamiltonian structure of the $n$-dimensional free rigid body equations. For any $f, g \in C^{\infty}(\operatorname{Sym}(n, N))$, the corresponding Lie-Poisson bracket is hence given by

$$
\{f, g\}_{N}(X):=-\operatorname{trace}\left(X[\nabla f(X), \nabla g(X)]_{N}\right)
$$

where $\nabla f$ is the gradient of $f$ on $\operatorname{Sym}(n, N)$ relative to the inner product $\left\langle\left\langle Y_{1}, Y_{2}\right\rangle:=\right.$ $\operatorname{trace}\left(Y_{1} Y_{2}\right)$, for any symmetric matrices $Y_{1}, Y_{2}$. The inner product $\langle\langle\cdot, \cdot\rangle\rangle$ is used to identify $\operatorname{Sym}(n, N)$ with its dual. The geometry, integrability, and linearizability of the flow of the 
Bloch-Iserles Hamiltonian system [5]

$$
\dot{X}=\left[X^{2}, N\right]=[X, X N+N X]
$$

on $\operatorname{Sym}(n, N)$ were investigated in [4], under the hypothesis that $N$ has distinct eigenvalues. The Hamiltonian function is $h(X)=\operatorname{trace}\left(X^{2} / 2\right)$ and (1.1) is equivalent to Hamilton's equations in Poisson bracket form $\dot{f}=\{f, h\}_{N}$ for any $f \in C^{\infty}(\operatorname{Sym}(n, N))$. An earlier study of the integrability of (1.1) from a different point of view can be found in [8].

In this paper, we continue the study of this Hamiltonian system by establishing its algebraic complete integrability, if $N$ has distinct eigenvalues. More precisely, we prove that the reduced Hamiltonian system (1.1) on the quotient space $\left(\lambda N+\operatorname{Sym}^{\mathbb{C}}(n, N)\right) / G_{0}$, where $G_{0}$ is a quotient group of the subgroup $G:=\mathbb{P} \mathrm{GL}_{n}(\mathbb{C} ; N)$ of the projective group $\mathbb{P G L}(\mathbb{C})$ formed by matrices which commute with $N$, is an algebraic completely integrable system, and, the Hamiltonian system (1.1) on the space $\lambda N+\operatorname{Sym}^{\mathbb{C}}(n, N)$ is a generalized algebraically completely integrable system; $\operatorname{Sym}^{\mathbb{C}}(n, N)$ is the complexification of $\operatorname{Sym}(n, N)$, that is, the space of complex symmetric matrices.

\section{Algebraic Integrability: Case $\mathfrak{g l}_{n}(\mathbb{C})$}

For the convenience of the reader we shall present shortly the known case $\mathfrak{g l}_{n}(\mathbb{C})$ (see $[3,7])$. We shall use the following definitions (compare with $[1,2,6,8]$ ).

Definition 2.1. Let $h: \mathcal{M} \rightarrow \mathcal{V}$ be a (complex) completely integrable system, where the Poisson manifold $\mathcal{M}$ is a nonsingular affine variety, $\mathcal{V}$ is an affine space, and $h=$ $\left(h_{1}, \ldots, h_{s}\right)$ is given by regular algebraic functions. We say that the system $h: \mathcal{M} \rightarrow \mathcal{V}$ is

(i) an algebraically completely integrable system (a.c.i. system) if each generic fiber of $h$ is a Zariski open subset of an Abelian variety, on which the Hamiltonian vector fields generated by $h_{i}$ are translation invariant

(ii) a generalized algebraically completely integrable system (generalized a.c.i. system) if each generic fiber of $h$ is a Zariski open subset of a commutative algebraic group, on which the Hamiltonian vector fields generated by $h_{i}$ are translation invariant.

The system (1.1) is equivalent to the following Lax pair system with parameter (see [4])

$$
\frac{\mathrm{d}}{\mathrm{d} t}(X+\lambda N)=\left[X+\lambda N, N X+X N+\lambda N^{2}\right] .
$$


We follow [3, 7] (see also [2, 6, 10]). From now on all Hamiltonian systems are complexified.

Let $X(\lambda):=\lambda N+X$, where $X \in \mathfrak{g l}_{n}(\mathbb{C})$ is an arbitrary matrix and $Q(\lambda, z):=$ $\operatorname{det}\left(z I_{n}-X(\lambda)\right)$ its characteristic polynomial. Denote by $M^{N}:=\lambda N+\mathfrak{g l}_{n}(\mathbb{C})=\{X(\lambda)=$ $\left.\lambda N+X \mid X \in \mathfrak{g l}_{n}(\mathbb{C})\right\}$ the affine space of all complex matrix polynomials of degree one whose leading coefficient is the constant real skew-symmetric matrix $N$. To stress the dependence on $N$ and $Q$ (both fixed), we denote the associated isospectral variety by $M_{Q}^{N}$, that is,

$$
M_{Q}^{N}:=\left\{X(\lambda) \in M^{N} \mid \operatorname{det}\left(z I_{n}-X(\lambda)\right)=Q(\lambda, z)\right\} .
$$

The plane algebraic curve (called a spectral curve), associated to each $X(\lambda)$, namely,

$$
\Gamma_{X(\lambda)}:=\left\{(\lambda, z) \in \mathbb{C} \times \mathbb{C} \mid \operatorname{det}\left(z I_{n}-X(\lambda)\right)=0\right\},
$$

is preserved by the flow of (2.1); the coefficients of the characteristic polynomial $Q(\lambda, z)$ of $X(\lambda)$ are polynomials which are constants of motion for the dynamics defined by (2.1). Similarly, for each $X(\lambda)$ the isospectral variety is preserved by the flow of (2.1). Notice that the spectral curve and the isospectral variety depend on the values of the constants of motion only (i.e., on the vector $\mathbf{c}=\left(q_{k l}\right)$, where $q_{k l}$ is the coefficient of $\lambda^{k} z^{l}$ in $Q(\lambda, z)$ ). Sometimes, one writes $\Gamma_{\mathrm{c}}$ instead of $\Gamma_{X(\lambda)}$. Notice that the spectral curve $\Gamma_{\mathrm{c}}$ is nonsingular for generic values of c. Let $\bar{\Gamma}_{\mathrm{c}}$ be the compactification in the projective plane $\mathbb{P}_{\mathbb{C}}^{2}$ of $\Gamma_{\mathbf{c}}$. For generic values of $\mathbf{c}$ the projective curve $\bar{\Gamma}_{\mathbf{c}}$ is also nonsingular. This is the case that we will consider.

The subgroup $G:=\mathbb{P} G L_{n}(\mathbb{C} ; N)$ of the projective group $\mathbb{P} G L_{n}(\mathbb{C})$ formed by matrices which commute with $N$ is a symmetry group of the system (2.1). Moreover, since $X+\lambda N$ commutes with its square, (2.1) can be written as the Lax equation with parameter

$$
\frac{\mathrm{d}}{\mathrm{d} t}(X+\lambda N)=\left[X^{2} / \lambda, X+\lambda N\right]
$$

This Lax equation (2.2) for an arbitrary matrix $X \in \mathfrak{g l}_{n}(\mathbb{C})$ was studied, for example, in $[3,7]$.

Let $\mathcal{V}$ be the affine space of polynomials

$$
Q(\lambda, z)=z^{n}+s_{1}(\lambda) z^{n-1}+\cdots+s_{n}(\lambda)
$$


where $s_{i}(\lambda)$ are polynomials in $\lambda$ of degree $\operatorname{deg} s_{i} \leq i$ for all $i=1, \ldots, n$. Consider the map

$$
h: M^{N} \rightarrow \mathcal{V},
$$

which sends a matrix of $M^{N}$ to its characteristic polynomial (the components of the map $h$ are the coefficients of the characteristic polynomial). Since $G$ acts freely and properly (by conjugation) on the affine space $M^{N}$, it follows that the quotient $M^{N} / G$ is a smooth variety. Moreover, we have the commutative diagram

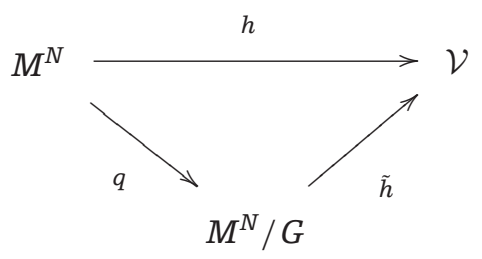

where $q: M^{N} \rightarrow M^{N} / G$ is the quotient map and $\tilde{h}: M^{N} / G \rightarrow \mathcal{V}$ is the map induced by $h$ (the action by conjugation preserves the characteristic polynomials).

Since $G$ acts freely and properly on the isospectral variety $M_{Q}^{N}$, it follows that $M_{Q}^{N}$ can be considered as the total space of a holomorphic principal fiber bundle with base space $M_{Q}^{N} / G$, structural group $G$, and natural projection map

$$
M_{Q}^{N} \longrightarrow M_{Q}^{N} / G
$$

Note that $M_{Q}^{N}$ is a fiber of $h$ and $M_{Q}^{N} / G$ is a fiber of $\tilde{h}$.

Generically, the spectral curve $\Gamma_{\mathrm{c}}$ (where $\mathbf{c}$ is the vector of the coefficients of the polynomial $Q$ ) is smooth. Then, the manifold $M_{Q}^{N} / G$ is bi-holomorphic to a Zariski open subset of the usual Jacobian $\operatorname{Jac}\left(\bar{\Gamma}_{\mathrm{c}}\right)$; see [3]. By [7, Theorem 2.1], the isospectral variety $M_{Q}^{N}$ is smooth and bi-holomorphic to a Zariski open subset of the generalized Jacobian variety $\operatorname{Jac}\left(\Gamma_{\mathrm{c}}^{\prime}\right)$, where $\Gamma_{\mathrm{c}}^{\prime}$ is the singular curve obtained from $\bar{\Gamma}_{\mathrm{c}}$ by identifying its points at infinity $\left\{P_{1}, \ldots, P_{n}\right\}$ with a single point $\infty$ (for details, see [11]).

The generalized Jacobian $\operatorname{Jac}\left(\Gamma_{\mathrm{c}}^{\prime}\right)$ is a noncompact commutative algebraic group given by a nontrivial extension of the usual Jacobian $\operatorname{Jac}\left(\bar{\Gamma}_{\mathrm{c}}\right)$ by the algebraic group $G=\mathbb{P} G L_{n}(\mathbb{C} ; N) \cong\left(\mathbb{C}^{*}\right)^{n-1}$, namely

$$
0 \longrightarrow G \longrightarrow \operatorname{Jac}\left(\Gamma_{\mathbf{c}}^{\prime}\right) \stackrel{\Phi}{\longrightarrow} \operatorname{Jac}\left(\bar{\Gamma}_{\mathbf{c}}\right) \longrightarrow 0 .
$$

The generalized Jacobian $\operatorname{Jac}\left(\Gamma_{\mathrm{c}}^{\prime}\right)$ can also be considered as total space of a holomorphic principal fiber bundle with base space $\operatorname{Jac}\left(\bar{\Gamma}_{\mathrm{c}}\right)$ and structure group $G$ and has dimension $g+n-1$, where $g$ is the genus of $\bar{\Gamma}_{\mathrm{c}}$. 
Beauville [3] and Gavrilov [7] proved that the Hamiltonian vector fields generated by the components of $\tilde{h}$, respectively of $h$, are translation invariant. We thus have the following results.

Theorem 2.1 ([3]). For $N$ having distinct eigenvalues, the system $\tilde{h}: M^{N} / G \rightarrow \mathcal{V}$ is an algebraically completely integrable system.

Theorem 2.2 ([7]). For $N$ having distinct eigenvalues, the system $h: M^{N} \rightarrow \mathcal{V}$ is a generalized algebraically completely integrable system.

\section{Algebraic Integrability: $\operatorname{Case} \operatorname{Sym}^{\mathbb{C}}(n, N)$}

Let us now consider the case of the Lie subalgebra

$$
\left.\operatorname{Sym}^{\mathbb{C}}(n, N),[,]_{N}\right) \subset\left(\mathfrak{g l}_{n}(\mathbb{C}),[,]_{N}\right)
$$

where $\mathfrak{g l}_{n}(\mathbb{C})$ is endowed with the bracket $[,]_{N}($ see [4]).

Let $M^{N, \text { Sym }}:=\lambda N+\operatorname{Sym}^{\mathbb{C}}(n, N)=\left\{X(\lambda):=\lambda N+X \mid X \in \operatorname{Sym}^{\mathbb{C}}(n, N)\right\}$ be the affine space of all complex matrix polynomials of degree one whose leading coefficient is the constant real skew-symmetric matrix $N$. We denote the associated isospectral variety by $M_{Q}^{N, \text { Sym }}$, that is,

$$
M_{Q}^{N, \text { Sym }}:=\left\{X(\lambda) \in M^{N, \text { Sym }} \mid \operatorname{det}\left(z I_{n}-X(\lambda)\right)=Q(\lambda, z)\right\}
$$

where $Q(\lambda, z)$ is the characteristic polynomial of $X(\lambda)$. We consider the generic case when the completion of the plane curve given by $Q=0$ is nonsingular.

We note that the affine subspace $M^{N \text {,Sym }}=\lambda N+\operatorname{Sym}^{\mathbb{C}}(n, N)$ is invariant by the automorphism (involution)

$$
\tau: \lambda N+\mathfrak{g l}_{n}(\mathbb{C}) \rightarrow \lambda N+\mathfrak{g l}_{n}(\mathbb{C}),
$$

given by $\tau(X(\lambda))=X^{\top}(-\lambda)$. Indeed, we have

$$
\tau(X(\lambda))=-\lambda N^{\top}+X^{\top}=\lambda N+X=X(\lambda),
$$

since $N^{\top}=-N$ and $X^{\top}=X$. 
Let $N$ be a given real skew-symmetric matrix. For $N$ invertible with distinct eigenvalues $(n:=2 p)$, choose an orthonormal basis of $\mathbb{R}^{2 p}$ in which $N$ is written as

$$
\left[\begin{array}{cc}
0 & V \\
-V & 0
\end{array}\right],
$$

where $V$ is a real diagonal matrix whose entries are $v_{1}, \ldots, v_{p}$.

For $N$ having distinct eigenvalues and nullity one, that is, $n:=2 p+1$ and rank $N=2 p$, choose an orthonormal basis of $\mathbb{R}^{2 p+1}$ in which $N$ is written as

$$
\left[\begin{array}{ccc}
0 & V & 0 \\
-V & 0 & 0 \\
0 & 0 & 0
\end{array}\right]
$$

where $V$ is a real diagonal matrix whose entries are $v_{1}, \ldots, v_{p}$.

Now, we compute explicitly the subgroup $G:=\mathbb{P} G L_{n}(\mathbb{C} ; N)$ of the projective group $\mathbb{P} G L_{n}(\mathbb{C})$ formed by matrices which commute with $N$. In Lemma 3.1, we use the form of $N$ given in (3.1).

Lemma 3.1. Let $n=2 p$ and $N$ invertible. Then, the group $\mathrm{GL}_{n}(\mathbb{C} ; N)$ of matrices which commute with $N$ is commutative and given by complex matrices $P$ of the form

$$
P=\left[\begin{array}{cc}
A & B \\
-B & A
\end{array}\right],
$$

where $A=\operatorname{diag}\left(a_{1}, \ldots, a_{p}\right)$ and $B=\operatorname{diag}\left(b_{1}, \ldots, b_{p}\right)$ are $\operatorname{diagonal}$ matrices and $\operatorname{det}(P) \neq 0$. Finally, $G:=\mathbb{P} \mathrm{GL}_{n}(\mathbb{C} ; N)=\mathrm{GL}_{n}(\mathbb{C} ; N) / \mathbb{C}^{\times}$, also a commutative group.

Proof. Write $P$ in the form

$$
\left[\begin{array}{ll}
A & B \\
C & D
\end{array}\right],
$$

and impose the condition $N P=P N$. Recalling that $N$ has the form (3.1), one gets the equalities:

$$
V C=-B V, \quad V D=A V, \quad V A=D V, \quad V B=-C V
$$

A direct computation, using that $V$ is diagonal and invertible, yields the result.

Similarly, using the form of $N$ given in (3.2), we get the following result. 
Lemma 3.2. Let $n=2 p+1, \operatorname{rank} N=2 p$, and $N$ has nullity one. Then, the group $\mathrm{GL}_{n}(\mathbb{C} ; N)$ of the matrices which commute with $N$ is commutative and given by complex matrices $P_{1}$ of the form

$$
P_{1}=\left[\begin{array}{ccc}
A & B & 0 \\
-B & A & 0 \\
0 & 0 & \alpha
\end{array}\right],
$$

where $A=\operatorname{diag}\left(a_{1}, \ldots, a_{p}\right)$ and $B=\operatorname{diag}\left(b_{1}, \ldots, b_{p}\right)$ are diagonal matrices and $\operatorname{det}\left(P_{1}\right) \neq 0$. Finally, $G:=\mathbb{P} \mathrm{GL}_{n}(\mathbb{C} ; N)=\mathrm{GL}_{n}(\mathbb{C} ; N) / \mathbb{C}^{\times}$, also a commutative group.

The affine subspace $M^{N \text {,Sym }}$ is not invariant by the action (by conjugation) of the commutative group $G$. We shall construct a quotient of the group $G$, which will act by conjugation on this affine subspace.

Firstly, remark that if $n=2 p$ and $T \in \mathrm{GL}_{n}(\mathbb{C} ; N)$ then

$$
T^{\top} T=\operatorname{diag}\left(d_{1}, \ldots, d_{p}, d_{1}, \ldots, d_{p}\right)
$$

where $d_{k}=a_{k}^{2}+b_{k}^{2}, k=1, \ldots, p$.

If $n=2 p+1$, then

$$
T^{\top} T=\operatorname{diag}\left(d_{1}, \ldots, d_{p}, d_{1}, \ldots, d_{p}, \alpha^{2}\right)
$$

Let $G_{1}$ be the subgroup of the group $G$ generated by the matrices of the form $\operatorname{diag}\left(d_{1}, \ldots, d_{p}, d_{1}, \ldots, d_{p}\right)$, where $d_{k} \in \mathbb{C}^{\times}, k=1, \ldots, p$, if $n=2 p$ and of the form $\operatorname{diag}\left(d_{1}, \ldots, d_{p}, d_{1}, \ldots, d_{p}, \alpha\right)$, where $d_{k} \in \mathbb{C}^{\times}, k=1, \ldots, p$, and $\alpha \in \mathbb{C}^{\times}$, if $n=2 p+1$. Denote the quotient $G / G_{1}$ by $G_{0}$ and observe that this quotient group $G_{0}$ is isomorphic to the subgroup of orthogonal matrices $\left(T^{\top} T=I_{n}\right)$ of the group $\mathrm{GL}_{n}(\mathbb{C} ; N)$.

Since $G_{0}$ acts freely and properly on the isospectral variety $M_{Q}^{N \text {,Sym }}$, it follows that $M_{Q}^{N, \text { Sym }}$ is the total space of a holomorphic principal bundle with base space $M_{Q}^{N \text {,Sym }} / G_{0}$, structural group $G_{0}$, and natural projection map

$$
\phi: M_{Q}^{N, \text { Sym }} \longrightarrow M_{Q}^{N, \text { Sym }} / G_{0}
$$

Recall that the bi-holomorphic map $l: M_{Q}^{N} / G \rightarrow U$, where $U$ is a Zariski open subset of the usual Jacobian $\operatorname{Jac}\left(\bar{\Gamma}_{\mathbf{c}}\right)$ (see $\left.[3,7]\right)$ is given by the eigenvector map. More precisely, let $f(\lambda, z)$ be a normalized eigenvector of the matrix $X(\lambda)$, where $(\lambda, z)$ is a point on the spectral curve $\bar{\Gamma}_{\mathbf{c}}$, with eigenvalue $z$. Then, it defines a line subbundle of the trivial vector bundle $\bar{\Gamma}_{\mathrm{c}} \times \mathbb{C}^{n}$, by taking at each point $(\lambda, z)$ of the spectral curve, the 
line along the normalized eigenvector $f(\lambda, z)$. Denote its dual by $L$; then $l(X(\lambda))=L$ (see [7, p. 495]).

By [7, Theorem 2.1], the isospectral variety $M_{Q}^{N}$ is smooth and bi-holomorphic to a Zariski open subset of the generalized Jacobian variety $\operatorname{Jac}\left(\Gamma_{\mathbf{c}}^{\prime}\right)$, where $\Gamma_{\mathbf{c}}^{\prime}$ is the singular curve obtained from $\bar{\Gamma}_{\mathrm{c}}$ by identifying its points at infinity $\left\{P_{1}, \ldots, P_{n}\right\}$ with a single point $\infty$. The bi-holomorphic map $l^{\prime}: M_{Q}^{N} \rightarrow U^{\prime}$, where $U^{\prime}$ is a Zariski open subset of the generalized Jacobian variety, is given by a similar eigenvector map (see [7]).

Since $(X+\lambda N)^{\top}=X-\lambda N$, we have $Q(-\lambda, z)=Q(\lambda, z)$, hence there is an involution

$$
\tau: \bar{\Gamma}_{\mathbf{c}} \rightarrow \bar{\Gamma}_{\mathbf{c}}
$$

of the spectral curve defined by $\tau(\lambda, z)=(-\lambda, z)$. The quotient smooth curve $C_{1}:=\bar{\Gamma}_{\mathbf{c}} / \tau$ has a double covering $\bar{\Gamma}_{\mathrm{c}} \rightarrow C_{1}$ and associated to this double covering is the Prym variety $\operatorname{Prym}\left(\bar{\Gamma}_{\mathbf{c}} / C_{1}\right)$, with the property that $\operatorname{Jac}\left(\bar{\Gamma}_{\mathbf{c}}\right)$ is isogenous to (see [4])

$$
\operatorname{Jac}\left(C_{1}\right) \times \operatorname{Prym}\left(\bar{\Gamma}_{\mathbf{c}} / C_{1}\right) .
$$

We have the following key result.

Lemma 3.3. The natural map $j: M_{Q}^{N, \text { Sym }} / G_{0} \rightarrow M_{Q}^{N} / G$ induced by the inclusion $M_{Q}^{N, \text { Sym } \hookrightarrow ~}$ $M_{Q}^{N}$ on the quotients is injective. The map $l \circ j: M_{Q}^{N, S y m} / G_{0} \rightarrow \operatorname{Prym}\left(\bar{\Gamma}_{\mathrm{c}} / C_{1}\right)$ is injective and maps bi-holomorphically $M_{Q}^{N, \text { Sym }} / G_{0}$ onto an open set of $\operatorname{Prym}\left(\bar{\Gamma}_{\mathbf{c}} / C_{1}\right)$.

Proof. The cases $n=2 p$ and $n=2 p+1$ are similar. The proof consists of several steps. In the third step, the two cases need to be considered separately.

Step 1 : The map $j$ is injective. It is easily verified that the inclusion $M_{Q}^{N, \text { Sym }} \hookrightarrow$ $M_{Q}^{N}$ drops to a map on the quotients $j: M_{Q}^{N, \text { Sym }} / G_{0} \rightarrow M_{Q}^{N} / G$.

Take two symmetric matrices $X_{1}, X_{2}$ such that the classes of the matrices $\lambda N+$ $X_{1}, \lambda N+X_{2}$ in $M_{Q}^{N, \text { Sym }} / G_{0}$ have the same image in $M_{Q}^{N} / G$ by the map $j$, that is, there exists a matrix $T \in G$ with $T X_{2}=X_{1} T$. Then $T$ has the form

$$
\left[\begin{array}{cc}
A & B \\
-B & A
\end{array}\right],
$$

where $A=\operatorname{diag}\left(a_{1}, \ldots, a_{p}\right)$ and $B=\operatorname{diag}\left(b_{1}, \ldots, b_{p}\right)$ are diagonal matrices, and $X_{1}, X_{2}$ have the form

$$
\left[\begin{array}{cc}
U_{i} & W_{i} \\
-W_{i}^{\top} & Q_{i}
\end{array}\right],
$$

$i=1,2$, with $U_{i}^{\top}=U_{i}$ and $Q_{i}^{\top}=Q_{i}$ 
A direct computation shows that the matrix

$$
F:=\left(A^{2}+B^{2}\right) U_{2}
$$

is symmetric. Denoting $d_{k}:=a_{k}^{2}+b_{k}^{2}, k=1, \ldots, p$, this relation implies

$$
d_{1}=d_{2}=\cdots=d_{p}=: s^{2}
$$

Thus, the matrix $T^{\prime}:=(1 / s) T \in G_{0}$ and has the property

$$
T^{\prime} X_{2}=X_{1} T^{\prime}
$$

which shows that the map $j$ is injective.

Step 2: If $X$ is a symmetric matrix, then l maps the class of $\lambda N+X$ in $M_{Q}^{N} / G$ to an element of $\operatorname{Prym}\left(\bar{\Gamma}_{c} / C_{1}\right)$, where $C_{1}:=\bar{\Gamma}_{c} / \tau$. For this purpose, we shall use [10, Sections 8 and 9].

As in [10, Section 8], we write for the equivalent Lax equations with parameter (2.3) and (2.4):

$$
M_{+}:=N X+X N+\lambda N^{2}, \quad M_{-}:=-X^{2} / \lambda,
$$

and we get

$$
M:=M_{+}-M_{-}=(X+\lambda N)^{2} / \lambda .
$$

Since $[X+\lambda N, M]=0$, it follows that the eigenvectors of $X+\lambda N$ are the also eigenvectors of $M$ (see [10, Theorem 8.3, p. 177]). Now, since the involution $\tau$ acts on $M$ by

$$
\tau(M)=-M,
$$

it follows for any eigenvalue $\mu$ of $M$, we have $\mu(\tau(P))=-\mu(P)$ for any point $P$ on the spectral curve $\Gamma_{\mathbf{c}}$ (see [10, p. 181]). By [10, Proposition 9.3], it follows that the line bundle, which is the image of $X+\lambda N$ by the map $l$, belongs to the anti-invariant part of the $\operatorname{Jacobian} \operatorname{Jac}\left(\bar{\Gamma}_{\mathbf{c}}\right)$, that is, it belongs to the Prym variety $\operatorname{Prym}\left(\bar{\Gamma}_{\mathbf{c}} / C_{1}\right)$.

Step 3: $\operatorname{dim}\left(M_{Q}^{N, \text { Sym }} / G_{0}\right)=\operatorname{dim}\left(\left(\operatorname{Prym}\left(\bar{\Gamma}_{c} / C_{1}\right)\right)\right.$. If $n=2 p$ we have the following computation of the dimensions of our manifolds. The dimension of the Jacobian $\operatorname{Jac}\left(\bar{\Gamma}_{\mathbf{c}}\right)$ is the genus $g$ of the spectral curve, which is

$$
g=(n-1)(n-2) / 2=2 p^{2}-3 p+1
$$


By the Riemann-Hurwitz formula, the curve $C_{1}$ has genus $g_{1}=(p-1)^{2}$ (see [4, p. 431]). It follows that

$$
\operatorname{dim}\left(\operatorname{Prym}\left(\bar{\Gamma}_{\mathbf{c}} / C_{1}\right)\right)=g-g_{1}=p^{2}-p
$$

Now, since a symmetric matrix has $2 p^{2}+p$ entries and the number of nonzero coefficients of the characteristic polynomials of a symmetric matrix is $p^{2}+p$, it follows that

$$
\operatorname{dim}\left(M_{Q}^{N, \text { Sym }}\right)=\left(2 p^{2}+p\right)-\left(p^{2}+p\right)=p^{2}
$$

and

$$
\operatorname{dim}\left(M_{Q}^{N, \text { Sym }} / G_{0}\right)=p^{2}-p=\operatorname{dim}\left(\operatorname{Prym}\left(\bar{\Gamma}_{\mathbf{c}} / C_{1}\right)\right)
$$

Similarly, if $n=2 p+1$ we get, $g=2 p^{2}-p, g_{1}=p^{2}-p$, and

$$
\operatorname{dim}\left(\operatorname{Prym}\left(\bar{\Gamma}_{\mathbf{c}} / C_{1}\right)\right)=g-g_{1}=p^{2} .
$$

Thus

$$
\operatorname{dim}\left(M_{Q}^{N, \text { Sym }}\right)=p^{2}+p
$$

and hence

$$
\operatorname{dim}\left(M_{Q}^{N, \text { Sym }} / G_{0}\right)=p^{2}=\operatorname{dim}\left(\operatorname{Prym}\left(\bar{\Gamma}_{\mathbf{c}} / C_{1}\right)\right)
$$

Step 4: $j \circ l: M_{Q}^{N, \text { Sym }} \rightarrow \operatorname{Prym}\left(\bar{\Gamma}_{c} / C_{1}\right)$ maps bi-holomorphically $M_{Q}^{N, \text { Sym }} / G_{0}$ onto an open set of $\operatorname{Prym}\left(\bar{\Gamma}_{c} / C_{1}\right)$. By the first two steps, $(j \circ l)\left(M_{Q}^{N, \operatorname{Sym}}\right) \subset \operatorname{Prym}\left(\bar{\Gamma}_{\mathbf{c}} / C_{1}\right)$ and $j \circ l$ is injective. By Step 3, $\operatorname{dim}\left(M_{Q}^{N, \text { Sym }} / G_{0}\right)=\operatorname{dim}\left(\operatorname{Prym}\left(\bar{\Gamma}_{\mathbf{c}} / C_{1}\right)\right)$. Thus [3, Theorem 1.4] yields the result.

Remark. The fact that the Hamiltonian vector fields are translation invariant follows by the results of Beauville [3] and Gavrilov [7] (see also [10]).

The previous lemma immediately implies the following result.

Theorem 3.1. For $N$ having distinct eigenvalues, the system $\tilde{h}_{0}: M^{N, \text { Sym }} / G_{0} \rightarrow \mathcal{V}_{\text {Sym }}$ is an algebraically completely integrable system.

Here, $\mathcal{V}_{\text {Sym }}$ consists of polynomials in $\mathcal{V}$ which have only even degrees in $\lambda$. 
The exact sequence (2.3) gives the following exact sequence of commutative groups:

$$
0 \longrightarrow G \stackrel{i}{\longrightarrow} \Phi^{-1}\left(\operatorname{Prym}\left(\bar{\Gamma}_{\mathbf{c}} / C_{1}\right)\right) \longrightarrow \operatorname{Prym}\left(\bar{\Gamma}_{\mathbf{c}} / C_{1}\right) \longrightarrow 0
$$

Let $\beta: G \rightarrow G_{0}$ be the quotient map and

$$
\eta: \operatorname{Ext}^{1}\left(\operatorname{Prym}\left(\bar{\Gamma}_{\mathbf{c}} / C_{1}\right), G\right) \longrightarrow \operatorname{Ext}^{1}\left(\operatorname{Prym}\left(\bar{\Gamma}_{\mathbf{c}} / C_{1}\right), G_{0}\right)
$$

the natural map of extensions induced by $\beta$.

Now we know that the extension (3.3) gives us the extension

$$
\eta\left(\Phi^{-1}\left(\operatorname{Prym}\left(\bar{\Gamma}_{\mathbf{c}} / C_{1}\right)\right)\right)
$$

of commutative groups

$$
0 \longrightarrow G_{0} \longrightarrow E \longrightarrow \operatorname{Prym}\left(\bar{\Gamma}_{\mathbf{c}} / C_{1}\right) \longrightarrow 0
$$

where

$$
E:=G_{0} \oplus \Phi^{-1}\left(\operatorname{Prym}\left(\bar{\Gamma}_{\mathrm{c}} / C_{1}\right)\right) / K
$$

and

$$
K:=\{(-\beta(g), i(g)) \mid g \in G\}
$$

Applying the result of Gavrilov [7] and a similar computation of dimensions, we get the following result.

Theorem 3.2. For $N$ having distinct eigenvalues, the system $h: M^{N, \text { Sym }} \rightarrow \mathcal{V}_{\text {Sym }}$ is a generalized algebraically completely integrable system.

Here, the generic fiber $M_{Q}^{N, S y m}$ is bi-holomorphic to a Zariski open set of the commutative algebraic group $E$.

\section{Funding}

V.B. was partially supported by a grant of the Romanian Ministry of National Education CNCSUEFISCDI, project number PN-II-ID-PCE-2012-4-0156 and by the Max-Planck-Institute für Mathematik, Bonn, Germany, where part of this paper was written. T.S.R. was partially supported by NCCR SwissMAP and grant 200021-140238, both of the Swiss National Science Foundation. 


\section{References}

[1] Adams, M. R., J. Harnad, and J. Hurturbise. "Darboux coordinates and Liouville-Arnold integration in loop algebras." Communications in Mathematical Physics 155, no. 2 (1993): 385-413.

[2] Adler, M. and P. van Moerbeke. "Linearization of Hamiltonian systems, Jacobi varieties and representation theory." Advances in Mathematics 38, no. 3 (1980): 318-79.

[3] Beauville, A. "Jacobiennes des courbes spectrales et systèmes hamiltoniens completement integrables." Acta Mathematica 164, no. 3-4 (1990): 211-35.

[4] Bloch, A. M., V. Brinzanescu, A. Iserles, J. E. Marsden, and T. S. Ratiu. "A class of integrable flows on the space of symmetric matrices." Communications in Mathematical Physics 290, no. 2 (2009): 399-435.

[5] Bloch, A. M. and A. Iserles. "On an isospectral Lie-Poisson system and its Lie algebra." Foundations of Computational Mathematics 6, no. 1 (2006): 121-44.

[6] Deift, P., L.-C. Li, and C. Tomei. "Matrix factorizations and integrable systems." Communications on Pure and Applied Mathematics XLII, no. 4 (1989): 443-521.

[7] Gavrilov, L. "Generalized Jacobians of spectral curves and completely integrable systems." Mathematische Zeitschrift 230, no. 3 (1999): 487-508.

[8] Li, L.-C. and C. Tomei. "The complete integrability of a Lie-Poisson system proposed by Bloch and Iserles." International Mathematics Research Notices (2006): Art. ID 64949. $19 \mathrm{pp}$.

[9] Morosi, C. and L. Pizzocchero. “On the Euler equation: bi-Hamiltonian structure and integrals in involution." Letters in Mathematical Physics 37, no. 2 (1996): 117-35.

[10] Reyman, A. G. and M. A. Semenov-Tian-Shansky. "Group Theoretical Methods in the Theory of Finite Dimensional Integrable Systems." Dynamical Systems VII, Enciclopedia of Mathematical Sciences 16. Berlin, Heidelberg, New York: Springer, 1994.

[11] Serre, J. P. Groupes Algebriques et Corps de Classes. Paris: Hermann, 1959.

[12] Trofimov, V. V. and A. T. Fomenko. "Noninvariant symplectic group structures and Hamiltonian flows on symmetric spaces." Trans. Sem. Vector Tensor Anal. 21 (1983): 23-83. English translation in Selecta Matematica Sovietica, 7, no. 4 (1988): 355-414. 